\title{
AN ALMOST NILPOTENT VARIETY OF EXPONENT 2
}

\author{
BY \\ S. MishCHENKO* \\ Department of Algebra and Geometric Computations \\ Ulyanovsk State University, Ulyanovsk 432970, Russia \\ e-mail: mishchenkosp@ulsu.ru
}

AND

A. VALEnTi**

Dipartimento di Ingegneria Elettrica, Elettronica e delle Telecomunicazioni di Tecnologie Chimiche, Automatica e Modelli Matematici

Università di Palermo, 90128 Palermo, Italy

e-mail: angela.valenti@unipa.it

\begin{abstract}
We construct a non-associative algebra $A$ over a field of characteristic zero with the following properties: if $\mathcal{V}$ is the variety generated by $A$, then $\mathcal{V}$ has exponential growth but any proper subvariety of $\mathcal{V}$ is nilpotent. Moreover, by studying the asymptotics of the sequence of codimensions of $A$ we deduce that $\exp (\mathcal{V})=2$.
\end{abstract}

\section{Introduction}

Let $F$ be a field of characteristic zero and $A$ a not necessarily associative $F$ algebra. A natural and well established way of measuring the polynomial identities satisfied by $A$ is through the study of the asymptotic behavior of its sequence of codimensions $c_{n}(A), n=1,2, \ldots$ In characteristic zero, without loss of generality, one can study the multilinear identities satisfied by $A$ and the sequence

\footnotetext{
* The first author was partially supported by RFBR grant 10-01-00209a.

** The second author was supported in part by MIUR of Italy. Received April 10, 2012 and in revised form October 1, 2012
} 
of codimensions actually gives a measure of such identities. More precisely, let $F\{X\}$ be the free non-associative algebra in a countable set $X$ and $P_{n}$ the space of multilinear polynomials in the first $n$ variables. If $\operatorname{Id}(A)$ denotes the T-ideal of polynomial identites satisfied by $A$, one considers the relatively free algebra $F\{X\} / \operatorname{Id}(A)$ and the sequence of subspaces $P_{n} /\left(P_{n} \cap \operatorname{Id}(A)\right), n \geq 1$. Then

$$
c_{n}(A)=\operatorname{dim} P_{n} /\left(P_{n} \cap I d(A)\right)
$$

is the $n$-th codimension of $A$

In the language of varieties, if $\mathcal{V}=\operatorname{var}(A)$ is the variety generated $A$, then the growth of $\mathcal{V}$ is the codimension growth of the algebra $A$. Also, we write $\operatorname{Id}(\mathcal{V})=\operatorname{Id}(A)$ and $c_{n}(\mathcal{V})=c_{n}(A)$.

The asymptotic behavior of the sequence of codimensions has been extensively studied in recent years leading to classification theorems (see, for instance, [7], [9], [14]). The key result in this area says that the sequence of codimensions of an associative PI-algebra (algebra satisfying a non-trivial polynomial identity) is exponentially bounded ([13]). Moreover, its exponential rate of growth, $\exp (A)=\lim _{n \rightarrow \infty} \sqrt[n]{c_{n}(A)}$, is an integer called the PI-exponent of $A$ ([4], [5], see also [7]).

For non-associative PI-algebras, the corresponding codimensions in general are not exponentially bounded. The same conclusion can be drawn for Lie and Jordan algebras. In case of Lie algebras an interesting scale of functions between exponential and factorial has been provided by Petrogradsky in [12]. Even when the codimensions are exponentially bounded, the exponential growth rate can be not an integer (see [11]). In fact in [6] the authors constructed for any real number $\alpha>1$ an algebra whose exponential growth of the codimensions is equal to $\alpha$.

Here we are interested in the largest T-ideals, or equivalently in the smallest varieties. Recall that an algebra $A$ is nilpotent if, for some $k \geq 1$, any product of $k$ elements of $A$ (with all possible arrangement of the brackets) is zero. Clearly if $A$ is a nilpotent algebra then $c_{n}(A)=0$, for $n$ large. Accordingly we say that a variety is nilpotent if it is generated by a nilpotent algebra. Hence nilpotent varieties are the ones of smallest growth. Clearly next smallest varieties must have all proper subvarieties nilpotent. A variety with such a property is called almost nilpotent. If we consider varieties of associative algebras, it is easily seen (see Section 3) that the only almost nilpotent variety is the variety $\mathcal{V}$ of commutative algebras (the sequence of codimensions is $c_{n}(\mathcal{V})=1, n \geq 1$ ). In 
case of varieties of Lie algebras, in [9] it was shown that there is also only one almost nilpotent variety: this is the metabelian variety, denoted $\mathcal{A}^{2}$, and in this case $c_{n}\left(\mathcal{A}^{2}\right)=n-1$.

In the non-associative case the situation is unclear. Anyway, by a standard application of Zorn's lemma, here we show that any variety contains a subvariety which is almost nilpotent.

Next we consider the problem of constructing an almost nilpotent variety of exponential growth and this is the main result of the paper. In fact we explicitly construct a non-associative algebra $A$ such that $\mathcal{V}=\operatorname{var}(A)$ is almost nilpotent. Moreover, we extensively study the structure of the multilinear identities of $A$ as a module of the symmetric group. We are able to determine almost all multiplicities in the $n$-th cocharacter of $\mathcal{V}$ and as a reward we prove that the variety $\mathcal{V}$ is almost nilpotent and $\exp (\mathcal{V})=\exp (A)$ exists and is equal to 2 .

\section{Preliminaries}

Throughout, $F$ will be a field of characteristic zero, $X=\left\{x_{1}, x_{2}, \ldots\right\}$ a countable set and $F\{X\}$ the free non-associative algebra on $X$ over $F$. For every $n \geq 1$, we consider $P_{n}$, the space of multilinear polynomials of $F\{X\}$ in the first $n$ variables $x_{1}, x_{2}, \ldots, x_{n}$. Notice that since the number of distinct arrangements of parentheses on a monomial of length $n$ is the Catalan number $\frac{1}{n}\left(\begin{array}{c}2 n-2 \\ n-1\end{array}\right)$, it readily follows that $\operatorname{dim}_{F} P_{n}=\left(\begin{array}{c}2 n-2 \\ n-1\end{array}\right)(n-1) !$.

Given an algebra $A$, let $I d(A)=\{f \in F\{X\} \mid f \equiv 0$ on $A\}$ be the T-ideal of $F\{X\}$ of polynomial identities of $A$. Since $\operatorname{char} F=0$, it is well known that the sequence of spaces $P_{n} \cap \operatorname{Id}(A), n=1,2, \ldots$, carries all information about $I d(A)$. The symmetric group $S_{n}$ acts on $P_{n}$ by permuting variables: if $\sigma \in S_{n}$, $f\left(x_{1}, \ldots, x_{n}\right) \in P_{n}$,

$$
\sigma f\left(x_{1}, \ldots, x_{n}\right)=f\left(x_{\sigma(1)}, \ldots, x_{\sigma(n)}\right) .
$$

The space $P_{n} \cap I d(A)$ is invariant under this action and one studies the structure of $P_{n}(A)=P_{n} /\left(P_{n} \cap I d(A)\right)$ as an $S_{n}$-module. The $S_{n}$-character of $P_{n}(A)$, denoted $\chi_{n}(A)$, is called the $n$th cocharacter of $A$. Its degree $c_{n}(A)=\chi_{n}(A)(1)$ is the $n$th codimension of $A$. By complete reducibility one writes $\chi_{n}(A)=$ $\sum_{\lambda \vdash n} m_{\lambda} \chi_{\lambda}$, where $\chi_{\lambda}$ is the irreducible $S_{n}$-character corresponding to the partition $\lambda$ of $n$ and $m_{\lambda} \geq 0$ is the multiplicity of $\chi_{\lambda}$ (see, for example, [8] for the representation theory of the symmetric group). 
If $\mathcal{V}=\operatorname{var}(A)$ is the variety generated by the algebra $A$, we define $P_{n}(\mathcal{V})=$ $P_{n}(A)$ and $\chi_{n}(\mathcal{V})=\chi_{n}(A)$.

In case $\mathcal{V}$ is a proper variety of associative algebras, by [1], the multiplicities $m_{\lambda}$ are polynomially bounded. If $A=F\langle X\rangle$ is the free associative algebra we have that in $\chi_{n}(A), m_{\lambda}=d_{\lambda}$ where $d_{\lambda}=\operatorname{deg} \chi_{\lambda}$ is the degree of the character $\chi_{\lambda}$. In the non-associative case these relations do not hold any more (see, for instance, [10], section 2). For instance, for the free non-associative algebra $A=F\{X\}$ we have that in $\chi_{n}(A), m_{\lambda}=C_{n} d_{\lambda}$ where $C_{n}$ is the $n$th Catalan number.

We next recall some basic properties of the representation theory of the symmetric group that we shall use in the sequel. Let $\lambda \vdash n$ be a partition and let $T_{\lambda}$ be a Young tableau of shape $\lambda \vdash n$. We denote by $e_{T_{\lambda}}$ the corresponding essential idempotent of the group algebra $F S_{n}$. Recall that $e_{T_{\lambda}}=\bar{R}_{T_{\lambda}} \bar{C}_{T_{\lambda}}$ where

$$
\bar{R}_{T_{\lambda}}=\sum_{\sigma \in R_{T_{\lambda}}} \sigma, \quad \bar{C}_{T_{\lambda}}=\sum_{\tau \in C_{T_{\lambda}}}(\operatorname{sgn} \tau) \tau
$$

and $R_{T_{\lambda}}, C_{T_{\lambda}}$ are the row and column stabilizers of $T_{\lambda}$, respectively. Recall that if $M_{\lambda}$ is an irreducible $S_{n}$-submodule of $P_{n}(\mathcal{V})$ corresponding to $\lambda$, then there exists a polynomial $f\left(x_{1}, \ldots, x_{n}\right) \in P_{n}$ and a tableau $T_{\lambda}$ such that $e_{T_{\lambda}} f\left(x_{1}, \ldots, x_{n}\right) \notin I d(\mathcal{V})$.

\section{Almost nilpotent subvarieties}

Let $\mathcal{V}$ be a variety of algebras. We say that $\mathcal{V}$ is a nilpotent variety if there exists $k \geq 1$ such that the product of $k$ variables (with all possible bracketing) is an identity of $\mathcal{V}$. Also, we say that a variety $\mathcal{V}$ is almost nilpotent if $\mathcal{V}$ is not a nilpotent variety but every proper subvariety $\mathcal{U} \subset \mathcal{V}$ is nilpotent.

We start this section by proving the following

Remark 1: Let $\mathcal{V}$ be the variety of associative commutative algebras. Then $\mathcal{V}$ is the only almost nilpotent variety of associative algebras.

Proof. Let $\mathcal{V}$ be the variety of associative commutative algebras. Then $\mathcal{V}$ can be generated by the algebra $A=F e$ where $e^{2}=e$. Let $\mathcal{U}$ be a variety of associative algebras and suppose that $\mathcal{V} \nsubseteq \mathcal{U}$. Then $\operatorname{Id}(\mathcal{U}) \nsubseteq I d(\mathcal{V})$ says that there exists a (multilinear) polynomial $f\left(x_{1}, \ldots, x_{k}\right) \in \operatorname{Id}(\mathcal{U}) \backslash \operatorname{Id}(\mathcal{V})$. 
Since $f \notin I d(\mathcal{V})=I d(A), f$ does not vanish on the basis $\{e\}$ of $A$. Thus if $f=\sum_{\sigma \in S_{k}} \alpha_{\sigma} x_{\sigma(1)} \cdots x_{\sigma(k)}$, we get $0 \neq f(e, \ldots, e)=\sum_{\sigma \in S_{k}} \alpha_{\sigma} e^{k}=\sum_{\sigma \in S_{k}} \alpha_{\sigma} e$. It follows that $\alpha=\sum_{\sigma \in S_{k}} \alpha_{\sigma} \neq 0$. Now let $\mathcal{U}=\operatorname{var}(B)$ and take any $b \in B$. Then $0=f(b, \ldots, b)=\alpha b^{k}$, which implies $b^{k}=0$. We have proved that if $\mathcal{U}$ is a variety of associative algebras and $\mathcal{V} \nsubseteq \mathcal{U}$, then $\mathcal{U}$ satisfies the identity $x^{k} \equiv 0$, for some $k \geq 1$. But then by the Nagata-Higman theorem (see [3]) $\mathcal{U}$ satisfies $x_{1} \cdots x_{t} \equiv 0$ for some $t \geq 1$, i.e., $\mathcal{U}$ is a nilpotent variety. This proves that any proper subvariety of $\mathcal{V}$ is nilpotent and $\mathcal{V}$ is the only almost nilpotent variety of associative algebras.

Theorem 1: Let $\mathcal{V}$ be a non-nilpotent variety of algebras. Then there exists a subvariety $\mathcal{W}$ of $\mathcal{V}$ such that $\mathcal{W}$ is almost nilpotent.

Proof. Let $S$ be the family of $T$-ideals $Q$ of $F\{X\}$ such that $Q \supseteq \operatorname{Id}(\mathcal{V})$ and $\frac{F\{X\}}{Q}$ is not nilpotent; $S$ is non-empty since it contains the $T$-ideal $\operatorname{Id}(\mathcal{V})$. We consider $S$ partially ordered by inclusion, and we let $\left\{Q_{i}\right\}_{i \in I}$ be a chain in $S$. If $Q=\bigcup_{i \in I} Q_{i}$, we claim that $Q \in S$, i.e., $Q \supseteq \operatorname{Id}(\mathcal{V})$ and $\frac{F\{X\}}{Q}$ is not nilpotent.

In fact if not, there exists $k \geq 1$ such that any multilinear monomial in $x_{1}, \ldots, x_{k}$ (with any arrangement of the brackets) belongs to $Q$. Let $C_{k}$ be the $k$-th Catalan number and let us denote by $\left\{w_{1}, \ldots, w_{C_{k}}\right\}$ the set of monomials obtained from $x_{1} \cdots x_{k}$ by placing the brackets in all possible ways. Then for every $i$, there exists $Q_{s_{i}}, s_{i} \in I$, such that $w_{i} \in Q_{s_{i}}$. If $s=\max _{i=1, \ldots, C_{k}} s_{i}$, then $w_{1}, \ldots, w_{C_{k}} \in Q_{s}$ and so $\frac{F\{X\}}{Q_{s}}$ is nilpotent, a contradiction.

By applying Zorn's lemma to $S$ it follows that there exists a maximal element $M \in S$. If $\mathcal{W}$ is the variety such that $\operatorname{Id}(\mathcal{W})=M$, then $\mathcal{W}$ is not nilpotent but any proper subvariety is nilpotent.

\section{The algebra $A$}

In this section we define a non-associative algebra $A$ that will be the main object of our investigation.

Let us denote by $R_{a}$ the operator of right multiplication by $a$.

Definition 1: $A$ is the algebra over $F$ generated by the elements $\{a, b, z\}$ satisfying the following relations:

(1) $a^{2}=b^{2}=a b=b a=a z=b z=0$; 
(2) $\left(z w\left(R_{a}, R_{b}\right)\right)\left(z w^{\prime}\left(R_{a}, R_{b}\right)\right)=0$, for any fixed eventually empty words $w, w^{\prime}$ in $R_{a}$ and $R_{b}$;

(3) for all $k \geq 0$,

$$
\begin{gathered}
z\left(R_{a} R_{b}\right)^{k} R_{a} R_{b}+z\left(R_{a} R_{b}\right)^{k} R_{b} R_{a}=0, \\
z\left(R_{a} R_{b}\right)^{k} R_{a}^{2}=z\left(R_{a} R_{b}\right)^{k} R_{b}^{2}=0 .
\end{gathered}
$$

The following lemma easily follows from the above relations.

Lemma 1: $A$ basis of $A$ is given by the elements

$$
a, b, z\left(R_{a} R_{b}\right)^{k}, z\left(R_{a} R_{b}\right)^{k} R_{a}, z\left(R_{a} R_{b}\right)^{k} R_{b},
$$

for all $k \geq 0$.

Proof. By the defining relations it is clear that the above elements span $A$. Next we prove that they are linearly independent over $F$. Let

(1) $\alpha_{1} a+\alpha_{2} b+\sum_{i} \beta_{i} z\left(R_{a} R_{b}\right)^{i}+\sum_{j} \gamma_{j} z\left(R_{a} R_{b}\right)^{j} R_{a}+\sum_{k} \delta_{k} z\left(R_{a} R_{b}\right)^{k} R_{b}=0$.

Applying the above on the right to $z$, we obtain that $\alpha_{1} z a+\alpha_{2} z b=0$. Hence $\alpha_{1} z a R_{a}+\alpha_{2} z b R_{a}=\alpha_{2} z b R_{a}=0$, and so $\alpha_{2}=\alpha_{1}=0$. If we now multiply (1) by $R_{a}$ on the right it follows that

$$
\sum_{i} \beta_{i} z\left(R_{a} R_{b}\right)^{i} R_{a}-\sum_{k} \delta_{k} z\left(R_{a} R_{b}\right)^{k+1}=0 .
$$

By multiplying again by $R_{a}$ on the right we obtain $\sum_{k} \delta_{k} z\left(R_{a} R_{b}\right)^{k+1} R_{a}=0$ and so $\delta_{k}=0, \forall k$. Similar calculations complete the proof.

The following remark will be of use in what follows.

Remark 2: Let $f\left(R_{a}, R_{b}\right)$ be a not necessarily homogeneous polynomial in $R_{a}, R_{b}$. If $z f\left(R_{a}, R_{b}\right) \neq 0$ then $z\left(R_{a} R_{b}\right) f\left(R_{a}, R_{b}\right) \neq 0$.

We next prove

Lemma 2: $x_{1}\left(x_{2} x_{3}\right)$ is an identity of $A$.

Proof. Notice that if $I$ is the ideal generated by $z$, then $\operatorname{dim} A / I=2$.

Let $\operatorname{ann}_{r}(A)=\{x \in A \mid A x=0\}$ be the right annihilator of $A$. Since $I \subseteq \operatorname{ann}_{r}(A)$ and, by definition of $A, A^{2} \subseteq I$, we obtain that $0=A I \supseteq A A^{2}$ and so $x_{1}\left(x_{2} x_{3}\right) \equiv 0$ holds on $A$. 
From Lemma 2 it is clear that only left normed polynomials can have a nonzero evaluation on $A$. Throughout we shall omit the parenthesis in left normed monomials.

LEMMA 3: The left normed monomial $x_{0} x x x$ is an identity of $A$.

Proof. Let $\varphi: F\{X\} \rightarrow A$ be an evaluation on $A$. Since $x_{0} x x x$ is linear in $x_{0}$ we may evaluate $x_{0}$ into a basis element of $A$.

If $\varphi\left(x_{0}\right)=a$ or $b$ then $\varphi(f)=0$.

Hence we assume that $\varphi\left(x_{0}\right)=z w\left(R_{a}, R_{b}\right)$, for some word $w$ in $R_{a}$ and $R_{b}$. Let $\varphi(x)=\alpha a+\beta b+c$, for some $\alpha, \beta \in F$ and $c \in I$, the ideal generated by $z$. Then, since $I \subseteq \operatorname{ann}_{r}(A)$, we may assume that $\varphi(x)=\alpha a+\beta b$. Now by direct inspection it is easily shown that $\varphi\left(x_{0} x x x\right)=0$. For instance, if $\varphi\left(x_{0}\right)=z\left(R_{a} R_{b}\right)^{k} R_{a}$,

$$
\begin{aligned}
\varphi\left(x_{0} x x x\right) & =z\left(R_{a} R_{b}\right)^{k} R_{a}\left(\alpha^{2} R_{a}^{2}+\beta^{2} R_{b}^{2}+\alpha \beta\left(R_{a} R_{b}+R_{b} R_{a}\right)\right)\left(\alpha R_{a}+\beta R_{b}\right) \\
& =\left(\beta^{2} z\left(R_{a} R_{b}\right)^{k+1} R_{b}+\alpha \beta z\left(R_{a} R_{b}\right)^{k+1} R_{a}\right)\left(\alpha R_{a}+\beta R_{b}\right) \\
& =\alpha \beta^{2} z\left(R_{a} R_{b}\right)^{k+1} R_{b} R_{a}+\alpha \beta^{2} z\left(R_{a} R_{b}\right)^{k+1} R_{a} R_{b}=0
\end{aligned}
$$

By applying a partial linearization to the identity of Lemma 3 we have the following

Remark 3: $x_{0} x_{1} x_{1} x_{2} \equiv-x_{0} x_{1} x_{2} x_{1}-x_{0} x_{2} x_{1} x_{1}(\bmod \operatorname{Id}(A))$.

LEMMA 4: $x_{0} x x y_{1} \cdots y_{2 s+1} y y$ is an identity of $A$.

Proof. Let $\varphi$ be an evaluation on $A$. By linearity on $x_{0}, y_{1} \cdots y_{2 s+1}$ and by what we remarked in the previous lemma, we may assume that $\varphi\left(x_{0}\right)=z w\left(R_{a}, R_{b}\right)$, for some $w, \varphi(x)=\alpha a+\beta b, \varphi(y)=\gamma a+\delta b$ and $\varphi\left(y_{i}\right)=\bar{y}_{i}=a$ or $b$, where $\alpha, \beta, \gamma, \delta \in F$.

If $\operatorname{deg} w=2 k$, then $w=\left(R_{a} R_{b}\right)^{k}$ and by the defining relations we get $\varphi\left(x_{0} x x y_{1} \cdots y_{2 s+1} y y\right)=z\left(R_{a} R_{b}\right)^{k}\left(\alpha^{2} R_{a}^{2}+\beta^{2} R_{b}^{2}+\alpha \beta\left(R_{a} R_{b}+R_{b} R_{a}\right)\right) \cdots=0$.

If $\operatorname{deg} w=2 k+1$, then either $w=\left(R_{a} R_{b}\right)^{k} R_{a}$ or $w=\left(R_{a} R_{b}\right)^{k} R_{b}$. In any case, by the defining relations,

$$
\varphi\left(x_{0} x x y_{1} \cdots y_{2 s+1} y y\right)=\mu z\left(R_{a} R_{b}\right)^{k+s+1}\left(\gamma^{2} R_{a}^{2}+\delta^{2} R_{b}^{2}+\gamma \delta\left(R_{a} R_{b}+R_{b} R_{a}\right)\right)=0
$$

for some $\mu \in F$, and the lemma is proved. 
From now on we shall write $X_{i}=R_{x_{i}}$ the right multiplication by the variable $x_{i}$.

Lemma 5: Let $w=w\left(X_{1}, X_{2}\right)$. If $\operatorname{deg}_{x_{1}} w-\operatorname{deg}_{x_{2}} w \geq 3$, then $x_{0} w\left(X_{1}, X_{2}\right) \equiv 0$ on $A$.

Proof. The proof is by induction on $k=\operatorname{deg}_{x_{2}} w$.

If $k=0$, we are done by Lemma 3 . Suppose that the conclusion of the lemma holds for $\operatorname{deg}_{x_{2}} w=k>0$ and let $\operatorname{deg}_{x_{2}} w=k+1$.

If $x_{0} w\left(X_{1}, X_{2}\right)$ is equal to $x_{0} X_{2} w^{\prime}$ or to $x_{0} X_{1} X_{2} w^{\prime}$, for some $w^{\prime}$, then since $\operatorname{deg}_{x_{2}} w^{\prime}<k+1$, we are done by induction.

Therefore we may assume that $x_{0} w\left(X_{1}, X_{2}\right)=x_{0} X_{1} X_{1} w^{\prime \prime}$, for some $w^{\prime \prime}$. Also, by Lemma 2 , we may assume that $x_{0} w\left(X_{1}, X_{2}\right)=x_{0} X_{1} X_{1} X_{2} w^{\prime \prime \prime}$, for some $w^{\prime \prime \prime}$. Now, by Remark 3, we can write

$$
x_{0} X_{1} X_{1} X_{2} \equiv-x_{0} X_{1} X_{2} X_{1}-x_{0} X_{2} X_{1} X_{1}(\bmod . I d(A)),
$$

and the proof follows from the previous cases.

\section{Some numerical invariants}

We start this section by proving some combinatorial propositions that will allow us to compute most multiplicities in the cocharacter of $\mathcal{V}=\operatorname{var}(A)$ for most partitions.

Recall that if $\lambda \vdash n$ is a partition of $n$ and $T_{\lambda}$ is a Young tableau of shape $\lambda$, we denote by $e_{T_{\lambda}} \in F S_{n}$ the corresponding essential idempotent of $F S_{n}$. In what follows we shall identify $e_{T_{\lambda}}$ with the polynomial $e_{T_{\lambda}}\left(y_{0} y_{1}, \ldots, y_{n}\right)=$ $y_{0} e_{T_{\lambda}} y_{1} \cdots y_{n}$ obtained by acting with $e_{T_{\lambda}}$ on the left normed monomial $y_{1} \cdots y_{n}$. We shall then identify all variables corresponding to each row of the tableau.

Let $Q_{n}=\operatorname{span}\left\{x_{0} x_{\sigma(1)} \cdots x_{\sigma(n)} \mid \sigma \in S_{n}\right\}$ be the space of multilinear left normed monomials in $x_{0}, \ldots, x_{n}$ with $x_{0}$ as the first variable. The symmetric group $S_{n}$ acts on $Q_{n}$ by permuting the variables $x_{1}, \ldots, x_{n}$ and $Q_{n} \cap \operatorname{Id}(A)$ is invariant under this action. Hence we have that

$$
Q_{n}(A)=\frac{Q_{n}}{Q_{n} \cap I d(A)}
$$

is an $S_{n}$-module and we denote by

$$
\chi_{n}^{Q}(A)=\chi^{S_{n}}\left(\frac{Q_{n}}{Q_{n} \cap I d(A)}\right)
$$


its character. We decompose $\chi_{n}^{Q}(A)$ into irreducible $S_{n}$-characters and we write

$$
\chi_{n}^{Q}(A)=\sum_{\lambda \vdash n} m_{\lambda}^{Q} \chi_{\lambda},
$$

where $m_{\lambda}^{Q}$ is the multiplicity of $\chi_{\lambda}$ in $\chi_{n}^{Q}(A)$.

Let $f \in Q_{n}$ and suppose that $f$ has a non-zero evaluation on $A$. Being multilinear $f$ has a non-zero evaluation on a basis of $A$. Hence we must evaluate $x_{0}$ into $z\left(R_{a} R_{b}\right)^{k}$ or $z\left(R_{a} R_{b}\right)^{k} R_{a}$ or $z\left(R_{a} R_{b}\right)^{k} R_{b}$. It follows that any other variable of $f$ must be evaluated into $a$ or $b$. As a consequence we get that any multilinear polynomial of $Q_{n}$ alternating on 3 variables is an identity of $A$. By standard arguments, this says that if the diagram of $\lambda \vdash n$ has more than two rows, then the corresponding $S_{n}$-character $\chi_{\lambda}$ appears with zero multiplicity in $\chi_{n}^{Q}(A)$. Also, let $\left(\lambda_{1}, \lambda_{2}\right) \vdash n$ be such that $\lambda_{1}-\lambda_{2} \geq 3$. Then for any tableau $T_{\lambda}$, if $f$ is the polynomial obtained from $e_{T_{\lambda}}\left(x_{0} x_{1}, \ldots, x_{n}\right)$ by identifying the variables corresponding to each row, we get that $\operatorname{deg}_{x_{1}} f-\operatorname{deg}_{x_{2}} f \geq 3$ and, by Lemma 5 , we obtain that $m_{\lambda}^{Q}=0$. Thus we obtain the decomposition

$$
\chi_{2 k}^{Q}(A)=m_{(k, k)}^{Q} \chi_{(k, k)}+m_{(k+1, k-1)}^{Q} \chi_{(k+1, k-1)}
$$

and

$$
\chi_{2 k+1}^{Q}(A)=m_{(k+1, k)}^{Q} \chi_{(k+1, k)} .
$$

In order to simplify the notation we shall also use the following convention: a monomial $M$ in which some variables are overlined by the same sign (say tilda, hat, etc.) must be read as the polynomial in which those variables are alternated.

We start by analyzing the partitions of the type $\lambda=(k+1, k-1), k \geq 1$. We have the following

Proposition 1: If $\lambda=(k+1, k-1), k \geq 1$, then $m_{(k+1, k-1)}^{Q}=1$.

Proof. Let $w_{1}, w_{2}$ be polynomials, obtained from the essential idempotents corresponding to two different tableaux of shape $\lambda$ by identifying the elements in each row of $\lambda$. Then $\operatorname{deg}_{x_{1}} w_{1}-\operatorname{deg}_{x_{2}} w_{1}=\operatorname{deg}_{x_{1}} w_{2}-\operatorname{deg}_{x_{2}} w_{2}=2$.

First we shall prove that $m_{(k+1, k-1)}^{Q} \leq 1$.

To this end we assume that $x_{0} w_{1}$ and $x_{0} w_{2}$ are not polynomial identities of $A$ and we need to prove that $x_{0} w_{1}$ and $x_{0} w_{2}$ are linearly dependent $(\bmod . \operatorname{Id}(A))$. We prove our claim by induction on $t=\operatorname{deg}_{x_{2}} w_{1}=\operatorname{deg}_{x_{2}} w_{2}$.

If $t=0$, then $w_{1}=w_{2}=x_{1} x_{1}$ and we are done. 
Let $\operatorname{deg}_{x_{2}} w=k>0$, where $w$ is a monomial, and suppose first that $x_{0} w=x_{0} X_{2} w^{\prime}$ for some $w^{\prime}$. Since $\operatorname{deg}_{x_{1}} w^{\prime}-\operatorname{deg}_{x_{2}} w^{\prime}=3$ then, by Lemma 5 , $x_{0} w \equiv 0(\bmod . I d(A))$. Therefore we must have that either $x_{0} w=x_{0} X_{1} X_{2} w^{\prime}$ or $x_{0} w=x_{0} X_{1} X_{1} w^{\prime \prime}$, for some $w^{\prime}, w^{\prime \prime}$. In the second case, from Lemma 3 , it follows that $x_{0} w=x_{0} X_{1} X_{1} X_{2} w^{\prime \prime \prime}$. Moreover, by Remark 3, we have that $x_{0} w=x_{0} X_{1} X_{1} X_{2} w^{\prime \prime \prime} \equiv-x_{0} X_{1} X_{2} X_{1} w^{\prime \prime \prime}(\bmod \operatorname{Id}(A))$.

Hence we may assume that $x_{0} w_{1}=x_{0} X_{1} X_{2} w_{1}^{\prime}$. Similar considerations concerning $w_{2}$ lead to $x_{0} w_{2}=x_{0} X_{1} X_{2} w_{2}^{\prime}$. But then we are done by induction. We have proved that $m_{(k+1, k-1)}^{Q} \leq 1$.

Next we prove that $m_{(k+1, k-1)}^{Q}=1$.

Let

$$
g_{k}=x_{0} x_{1} x_{1} \underbrace{\overline{x_{1}} \overline{x_{2}} \cdots \hat{x_{1}} \hat{x_{2}}}_{k-1 \text { pairs }}
$$

be the left-normed polynomial associated to $\lambda=(k+1, k-1)$, where ${ }^{-\wedge}$ mean alternation on the corresponding elements. Notice that the polynomial $g_{k}$ is obtained from the essential idempotent corresponding to $\lambda$ by identifying all the elements in each row of $\lambda$.

We claim that if $\varphi$ is an evaluation on $A$ such that $\varphi\left(x_{0}\right)=z a, \varphi\left(x_{1}\right)=$ $a+b, \varphi\left(x_{2}\right)=b$, then $\varphi\left(g_{k}\right)=(-1)^{k-1} z(a b)^{k}(a+b) \neq 0$.

In fact, by induction on $k$, if $k=1$ then $\varphi\left(g_{1}\right)=\varphi\left(x_{0} x_{1} x_{1}\right)=z a b(a+b)$. Now, in general,

$$
\varphi\left(g_{k+1}\right)=\varphi\left(g_{k}\right) \widehat{(a+b)} \widehat{b}=(-1)^{k-1} z(a b)^{k}(a+b) \widehat{(a+b)} \widehat{b} .
$$

Since by (3) of Definition 1

$$
z(a b)^{k}(a+b)(a+b) b \equiv 0(\bmod . \operatorname{Id}(A)),
$$

it follows that $\varphi\left(g_{k+1}\right)=(-1)^{k} z(a b)^{k+1}(a+b) \neq 0$, and we are done.

Proposition 2: If $\lambda=(k+1, k)$ then $m_{(k+1, k)}^{Q}=2$.

Proof. By induction on $k$. If $k=1$, since $d_{(2,1)}=\operatorname{deg} \chi_{(2,1)}=2$, we are done.

Let $k \geq 2$, and let $w=w\left(x_{1}, x_{2}\right)$ be a homogeneous polynomial such that $\operatorname{deg}_{x_{1}} w-\operatorname{deg}_{x_{2}} w=1$. We claim that $x_{0} w$ can be written as a linear combination of polynomials of the type $x_{0} X_{2} w^{\prime}$ and $x_{0} w^{\prime} X_{2}$ for some $w^{\prime}$.

In fact, if we apply partial linearization to the identity of Lemma 3 we obtain

$$
x_{0} X_{1} X_{1} X_{2} \equiv-x_{0} X_{1} X_{2} X_{1}-x_{0} X_{2} X_{1} X_{1}(\bmod . \operatorname{Id}(A))
$$


and

$$
x_{0} u X_{2} X_{1} X_{1} \equiv-x_{0} u X_{1} X_{2} X_{1}-x_{0} u X_{1} X_{1} X_{2}(\bmod . \operatorname{Id}(A)),
$$

for any monomial $u$. It follows that $x_{0} w$ is a linear combination of polynomials of the type $x_{0} X_{2} w^{\prime}, x_{0} w^{\prime} X_{2}$ and $x_{0} X_{1} X_{2} v X_{2} X_{1}$ for some $w^{\prime}, v$ with deg $v=2 k-3$.

But linearizing the identity of Lemma 4 , for any $v$ with $\operatorname{deg} v=2 k-3$ we get

$$
x_{0} X_{1} X_{2} v X_{2} X_{1} \equiv-x_{0} X_{2} X_{1} v X_{1} X_{2}-x_{0} X_{1} X_{2} v X_{1} X_{2}-x_{0} X_{2} X_{1} v X_{2} X_{1}
$$

and the claim is proved.

Let $H_{k+1, k-1}$ be the space of homogeneous polynomials in $x_{1}$, and $x_{2}$ of degree $k+1$ in $x_{1}$ and $k-1$ in $x_{2}$. Then Proposition 1 says that $\operatorname{dim} x_{0} H_{k+1, k-1} \leq 1$ and $\operatorname{dim} x_{0} H_{k+1, k-1} X_{2} \leq 1(\bmod . \operatorname{Id}(A))$. Hence from the previous claim we get that $m_{(k+1, k)}^{Q} \leq 2$.

Define the following two polynomials:

$$
g_{1}=x_{0} x_{1} \overline{x_{1}} \overline{x_{2}} \cdots \widetilde{x_{1}} \widetilde{x_{2}}=x_{0} g_{1}^{\prime}
$$

and

$$
g_{2}=x_{0} \overline{x_{1}} \overline{x_{2}} \cdots \widetilde{x_{1}} \widetilde{x_{2}} x_{1}=x_{0} g_{2}^{\prime} .
$$

The polynomials $g_{1}^{\prime}, g_{2}^{\prime}$ are obtained from the essential idempotents corresponding to the tableaux

$$
T_{\lambda}^{1}=\begin{array}{|c|c|c|c|c|}
\hline 2 & 4 & \cdots & n-1 & 1 \\
\hline 3 & 5 & \cdots & n
\end{array} \quad \text { and } T_{\lambda}^{2}=\begin{array}{|l|l|l|l|l|}
\hline 1 & 3 & \cdots & n-2 & n \\
\hline 2 & 4 & \cdots & n-1 &
\end{array}
$$

by identifying the integers in each row of $\lambda$.

In order to complete the proof of the proposition it is enough to prove that $g_{1}, g_{2}$ are linearly independent $(\bmod . \operatorname{Id}(A))$. Write $g=\alpha g_{1}+\beta g_{2} \equiv 0$ $(\bmod \operatorname{Id}(A))$, for some $\alpha, \beta \in F$.

Let $\varphi$ be an evaluation on $A$ such that $\varphi\left(x_{1}\right)=a, \varphi\left(x_{2}\right)=b, \varphi\left(x_{0}\right)=z$.

Since by the defining relation on $A$,

$$
z\left(R_{a} R_{b}\right)^{k} R_{a} R_{b} \equiv-z\left(R_{a} R_{b}\right)^{k} R_{b} R_{a}(\bmod . \operatorname{Id}(A)),
$$

and by Remark 3,

$$
z a b a \equiv-z a a b-z b a a \equiv-z b a a(\bmod . \operatorname{Id}(A)),
$$

we obtain

$$
\varphi(g)=(-1)^{k} \alpha z a b a b \cdots a b a+2^{k} \beta z a b a b \cdots a b a \equiv 0(\bmod . \operatorname{Id}(A))
$$


and so

$$
(-1)^{k} \alpha+2^{k} \beta=0 \text {. }
$$

In the same way, by putting $\varphi\left(x_{1}\right)=a, \varphi\left(x_{2}\right)=b, \varphi\left(x_{0}\right)=z b$ we have

$$
\left(2^{k} \alpha+(-1)^{k} \beta\right) z b a b a b \cdots a b \equiv 0(\bmod . \operatorname{Id}(A)) .
$$

It follows that

$$
2^{k} \alpha+(-1)^{k} \beta=0
$$

Hence from the above we have that $\alpha=\beta=0$, and this implies that $m_{(k+1, k)}^{Q}=$ 2 .

Proposition 3: If $\lambda=(k, k)$ then $0<m_{(k, k)}^{Q} \leq 2$.

Proof. First we prove that $m_{(k, k)}^{Q}>0$. Let $n=2 k$ and define the following polynomial:

$$
g=x_{0} \overline{x_{1}} \overline{x_{2}} \cdots \widetilde{x_{1}} \widetilde{x_{2}}=x_{0} g^{\prime},
$$

where we have $k$ pairs $x_{1} x_{2}$.

The polynomial $g^{\prime}$ is obtained from the essential idempotent corresponding to the tableaux

$$
T_{\lambda}=\begin{array}{|l|l|l|c|}
\hline 1 & 3 & \cdots & n-1 \\
\hline 2 & 4 & \cdots & n \\
\hline
\end{array}
$$

by identifying the integers in each row. If $m_{(k, k)}=0$ then $g \equiv 0(\bmod . \operatorname{Id}(A))$. From this it follows that $g_{1} \equiv 0$ and $g_{2} \equiv 0$ (mod. $\left.I d(A)\right)$, where $g_{1}$ and $g_{2}$ are the polynomials defined in the proof of Proposition 2, a contradiction. Hence $m_{(k, k)}^{Q}>0$.

Let $w$ be a polynomial in $x_{1}$ and $x_{2}$, with $\operatorname{deg}_{x_{1}} w=\operatorname{deg}_{x_{2}} w=k$. By using a partial linearization of the identity in Lemma 3, it easily follows that mod. $I d(A), w$ can be written as a linear combination of monomials of the type

for some $w^{\prime}, w^{\prime \prime}, w^{\prime \prime \prime}, v$.

$$
x_{1} x_{2} x_{2} w^{\prime}, x_{2} x_{1} x_{1} w^{\prime \prime}, x_{1} x_{1} x_{2} w^{\prime \prime \prime}, x_{2} x_{2} x_{1} v,
$$

By a repeated application of this argument we get the following.

If $k=2 m$, then $w$ can be written as a linear combination of the following 4 monomials,

$$
\begin{aligned}
& w_{1}=x_{1} x_{1} x_{2} x_{2} \cdots x_{1} x_{1} x_{2} x_{2}, \\
& w_{1}^{\prime}=x_{2} x_{2} x_{1} x_{1} \cdots x_{2} x_{2} x_{1} x_{1}, \\
& w_{2}=x_{1} x_{2} x_{2} x_{1} \cdots x_{1} x_{2} x_{2} x_{1}, \\
& w_{2}^{\prime}=x_{2} x_{1} x_{2} x_{1} x_{2} \cdots x_{2} x_{1} x_{1} x_{2} .
\end{aligned}
$$


If $k=2 m+1$, then $w$ can be written as a linear combination of the following monomials:

$$
\begin{aligned}
& \bar{w}_{1}=x_{1} x_{1} x_{2} x_{2} \cdots x_{1} x_{1} x_{2} x_{2} x_{1} x_{2}, \\
& \bar{w}_{1}^{\prime}=x_{2} x_{2} x_{1} x_{1} \cdots x_{2} x_{2} x_{1} x_{1} x_{2} x_{1}, \\
& \bar{w}_{2}=x_{1} x_{2} x_{2} x_{1} \cdots x_{1} x_{2} x_{2} x_{1} x_{1} x_{2}, \\
& \bar{w}_{2}^{\prime}=x_{2} x_{1} x_{1} x_{1} x_{2} \cdots x_{2} x_{1} x_{1} x_{2} x_{2} x_{1} .
\end{aligned}
$$

Let $g$ be a highest weight vector corresponding to the partition $\lambda=(k, k)$. If $k=2 m$, then we can write $g \equiv \alpha w_{1}+\beta w_{1}^{\prime}+\gamma w_{2}+\delta w_{2}^{\prime}$, for some $\alpha, \beta, \gamma, \delta \in F$. Since $g$ is alternating on $k$ pairs $\left\{x_{1}, x_{2}\right\}$, we get that

$$
g\left(x_{1}, x_{2}\right) \equiv(-1)^{2 m} g\left(x_{2}, x_{1}\right)=\alpha w_{1}^{\prime}+\beta w_{1}+\gamma w_{2}^{\prime}+\delta w_{2} .
$$

Hence

$$
g \equiv \frac{\alpha+\beta}{2}\left(w_{1}+w_{1}^{\prime}\right)+\frac{\gamma+\delta}{2}\left(w_{2}+w_{2}^{\prime}\right)(\bmod . \operatorname{Id}(A)) .
$$

It follows that any highest weight vector corresponding to the partition $\lambda=$ $(k, k)$ is a linear combination of $\left(w_{1}+w_{2}\right)$ and $\left(w_{1}^{\prime}+w_{2}^{\prime}\right)$ and so $m_{(k, k)}^{Q} \leq 2$.

Similarly, if $k=2 m+1$, write

$$
g\left(x_{1}, x_{2}\right) \equiv \alpha \bar{w}_{1}+\beta \bar{w}_{1}^{\prime}+\gamma \bar{w}_{2}+\delta \bar{w}_{2}^{\prime}
$$

and we get

$$
g\left(x_{1}, x_{2}\right) \equiv(-1)^{2 m+1} g\left(x_{2}, x_{1}\right)=\alpha \bar{w}_{1}^{\prime}+\beta \bar{w}_{1}+\gamma \bar{w}_{2}^{\prime}+\delta \bar{w}_{2} .
$$

Hence

$$
g \equiv \frac{\alpha-\beta}{2}\left(w_{1}-w_{1}^{\prime}\right)+\frac{\gamma-\delta}{2}\left(w_{2}-w_{2}^{\prime}\right)(\bmod . \operatorname{Id}(A)) .
$$

As above this says that $m_{(k, k)}^{Q} \leq 2$, and the proof is complete.

Putting together the results so far obtained, we have the following

THEOREM 2: $\quad(1) \chi_{2 k+1}^{Q}(A)=2 \chi_{(k+1, k)}$.

(2) $\chi_{2 k}^{Q}(A)=a \chi_{(k, k)}+\chi_{(k+1, k-1)}$ with $a=1$ or $a=2$.

In the next proposition we shall determine the relation between the sequence of codimensions $c_{n}^{Q}(A)$ and $c_{n}(A), n=1,2, \ldots$

Proposition 4: For all $n \geq 1$ we have that $c_{n+1}(A)=(n+1) \cdot c_{n}^{Q}(A)$. 
Proof. For every $i \in\{0, \ldots, n\}$ let us denote by $Q_{n}^{i}$ the vector space spanned by the left normed monomials in $x_{0}, x_{1}, \ldots, x_{n}$ starting with $x_{i}$. Hence in this terminology $Q_{n}=Q_{n}^{0}$. Let us also denote by $P_{n+1}(A)$ the space of multilinear left normed polynomials in $x_{0}, x_{1}, \ldots, x_{n}$ (not in $x_{1}, x_{2}, \ldots, x_{n+1}$ as before). It is obvious that

$$
P_{n+1}(A)=Q_{n}^{0}(A)+\cdots+Q_{n}^{n}(A),
$$

and so $c_{n+1}(A) \leq(n+1) \cdot c_{n}^{Q}(A)$.

We claim that

$$
P_{n+1}(A)=Q_{n}^{0}(A) \oplus \cdots \oplus Q_{n}^{n}(A) .
$$

This is clear by the structure of the algebra $A$. We only note that if $x_{0} f_{1}\left(x_{1}, \ldots, x_{n}\right), \ldots, x_{0} f_{m}\left(x_{1}, \ldots, x_{n}\right) \in Q_{n}^{0}$ are linearly independent (mod. $I d(A))$, then the $(n+1) \cdot m$ left-normed polynomials of $P_{n+1}$

$$
x_{0} f_{i}\left(\widehat{x_{0}}, x_{1}, \ldots, x_{n}\right), \ldots, x_{n} f_{i}\left(x_{0}, x_{1}, \ldots, \widehat{x_{n}}\right), \quad i=1, \ldots, m,
$$

are linearly independent $(\bmod . \operatorname{Id}(A))$, where ${ }^{\wedge}$ means that the corresponding element is omitted.

Next we want to study the variety of algebras $\mathcal{V}=\operatorname{var}(A)$, generated by $A$. Our aim is to prove that $\mathcal{V}$ is almost nilpotent. We start by proving the following

Proposition 5: Let $\mathcal{W} \varsubsetneqq \mathcal{V}$ be a proper subvariety of $\mathcal{V}$. If, for some $n \geq 1$, $x_{0} \underbrace{x_{1} x_{1} x_{2} x_{2} x_{1} x_{1} \cdots x_{2} x_{2} x_{1} x_{1}}_{2 n} \in I d(\mathcal{W})$, then $c_{N}^{Q}(\mathcal{W})=0$, for all $N \geq 2 n+2$.

Proof. Clearly $c_{N}^{Q}(\mathcal{W})=0$ means that $Q_{N} \subseteq I d(\mathcal{W})$. Now since $\mathcal{W} \varsubsetneqq \mathcal{V}$, recalling the decomposition given in Theorem 2, we get that $\chi_{n}^{Q}(\mathcal{W})<\chi_{n}^{Q}(\mathcal{V})$, i.e., $\chi_{2 k+1}^{Q}(\mathcal{W})=a \chi_{(k+1, k)}$ and $\chi_{2 k}^{Q}(\mathcal{W})=b \chi_{(k, k)}+c \chi_{(k+1, k-1)}$, with $a \leq 2$, $b \leq 2, c \leq 1$. In particular, this says that in order to prove that $c_{N}^{Q}(\mathcal{W})=0$ it is enough to show that $x_{0} H_{h, l} \subseteq I d(\mathcal{W})$, where $H_{h, l}$ is the space of homogeneous polynomials of degree $h$ in $x_{1}$ and $l$ in $x_{2}$. Here $l=k$ or $l=k-1$ or $l=k-2$. Let $v=x_{0} w\left(x_{1}, x_{2}\right) \in x_{0} H_{k, l}(\bmod . \operatorname{Id}(\mathcal{W}))$.

We claim that $v=x_{0} w\left(x_{1}, x_{2}\right) \equiv x_{0} x_{1} x_{1} w^{\prime}$ or $x_{0} x_{2} x_{2} w^{\prime}$ or $x_{0} x_{1} x_{2} x_{2} w^{\prime}$ or $x_{0} x_{2} x_{1} x_{1} w^{\prime}$, for some $w^{\prime}$.

In fact, if $v=x_{0} x_{1} x_{1} v^{\prime}$ or $x_{0} x_{2} x_{2} w^{\prime}$ for some $v^{\prime}, w^{\prime}$, we are done.

Otherwise, recalling that, by Remark $3, x_{0} x_{1} x_{2} x_{1} \equiv-x_{0} x_{2} x_{1} x_{1}-x_{0} x_{1} x_{1} x_{2}$, we get the claim. Now, by eventually exchanging the role of $x_{1}$ and $x_{2}$, we may 
assume that $v=u x_{1} x_{1} w^{\prime}$ where $u=x_{0}$ or $u=x_{0} x_{2}$ and $w^{\prime}$ is a polynomial, not necessarily homogeneous, in $x_{1}$ and $x_{2}$. Then since $x_{0} x_{1} x_{1} x_{1} \equiv 0$, it follows that

$$
v=u x_{1} x_{1} x_{2} x_{2} x_{1} w^{\prime \prime}
$$

or

$$
v=u x_{1} x_{1} x_{2} x_{1} x_{2} w^{\prime \prime \prime} \equiv-u x_{1} x_{1} x_{2} x_{2} x_{1} w^{\prime \prime \prime} .
$$

Now the proof is complete by induction on $n$. Notice that in this construction we have eventually one extra variable at the beginning after $x_{0}$ and eventually one extra variable at the right-end side.

We can now prove the main result of the paper.

ThEOREM 3: The variety $\mathcal{V}=\operatorname{var}(A)$ is almost nilpotent and $\exp (\mathcal{V})=$ $\exp (A)=2$.

Proof. We first show that $\exp (A)=2$.

In fact, from the decomposition of the cocharacter of $Q_{n}(A)$ and Proposition 4 we have the asymptotic equality $\sqrt[n]{c_{n}(A)} \simeq \sqrt[n]{d_{(m, m)}}$, where $n=2 m$ or $2 m+1$. Since $\lim _{n \rightarrow \infty} \sqrt[n]{d_{(m, m)}}=2$, we get that $\exp (A)=2$.

Next we show that $\mathcal{V}=\operatorname{var}(A)$ is almost nilpotent.

Let $\mathcal{W} \varsubsetneqq \mathcal{V}$ be a proper subvariety. Then, there exists a multilinear polynomial $f$, of degree $l$, such that $f \in \operatorname{Id}(\mathcal{W})$ and $f \notin I d(\mathcal{V})$. We claim that we may assume that $f=x_{0} w$ where $w=w\left(R_{x_{1}}, R_{x_{2}}\right)$. In fact, since $x(y z) \in \operatorname{Id}(\mathcal{V})$, we have that $f=\sum x_{s} w_{s}$ with $w_{s}=w_{s}\left(R_{x_{1}}, \ldots, \widehat{R_{x_{s}}}, \ldots, R_{x_{l}}\right)$ (here again, the symbol $^{\wedge}$ means that the corresponding element is omitted).

Let $t$ be such that $x_{t} w_{t} \notin I d(\mathcal{V})$ and let $\varphi$ be a non-zero evaluation in $A$. Since $f$ is linear, by Lemma 1 , we may assume that $\varphi\left(x_{t}\right)=z\left(R_{a} R_{b}\right)^{k}$ or $z\left(R_{a} R_{b}\right)^{k} R_{a}$ or $z\left(R_{a} R_{b}\right)^{k} R_{b}$ and $k \geq 1$. If we now make the substitution $x_{t}=x_{0} x_{l+1} x_{l+2}$ in $f$, then we obtain $x_{0} x_{l+1} x_{l+2} w_{t} \notin I d(\mathcal{V})$ and $x_{0} x_{l+1} x_{l+2} w_{t} \in \operatorname{Id}(\mathcal{W})$.

In fact if $\varphi\left(x_{t}\right)=z\left(R_{a} R_{b}\right)^{k}$, it is sufficient to set $x_{0}=\varphi\left(x_{t}\right)=z\left(R_{a} R_{b}\right)^{k-1}$, $x_{l+1}=R_{a}, x_{l+2}=R_{b}$, similarly for the other cases. Since $\chi_{n}^{Q}(A)$ lies in a strip of height 2 , we get the claim, i.e., we may assume that $f=x_{0} w$ and $w$ is in only two variables $x_{1}$ and $x_{2}$.

By multiplying on the right by $x_{1}$ and $x_{2}$ in a suitable way, we may assume that $f=x_{0} w$ and $\operatorname{deg} w=4 m+1$. By Proposition 2 or its proof we may assume that

$$
f=\alpha x_{0} x_{1} \underbrace{\overline{x_{1}} \overline{x_{2}} \cdots \tilde{x_{1}} \tilde{x_{2}}}_{2 m \text { pairs }}+\beta x_{0} \underbrace{\overline{x_{1}} \overline{x_{2}} \cdots \tilde{x_{1}} \tilde{x_{2}}}_{2 m \text { pairs }} x_{1} \in I d(\mathcal{W})
$$


with $\alpha \beta \neq 0$. If we now substitute $x_{0} x_{1}$ for $x_{0}$, we get

$$
\alpha x_{0} x_{1} x_{1} \overline{x_{1}} \overline{x_{2}} \cdots \tilde{x_{1}} \tilde{x_{2}}+\beta x_{0} x_{1} \overline{x_{1}} \overline{x_{2}} \cdots \tilde{x_{1}} \tilde{x_{2}} x_{1} \in \operatorname{Id}(\mathcal{W}) .
$$

Multiplying on the right by $x_{1}$ we obtain

$$
\alpha x_{0} x_{1} \overline{x_{1}} \overline{x_{2}} \cdots \tilde{x_{1}} \tilde{x_{2}} x_{1}+\beta x_{0} \overline{x_{1}} \overline{x_{2}} \cdots \tilde{x_{1}} \tilde{x_{2}} x_{1} x_{1} \in I d(\mathcal{W}) .
$$

Set

$$
g_{0}=x_{0} \underbrace{x_{1} x_{1} x_{2} x_{2} \ldots x_{1} x_{1} x_{2} x_{2}}_{4 m} x_{1} x_{1}, \quad g_{1}=x_{0} x_{1} x_{1} \underbrace{\overline{x_{1}} \overline{x_{2}} \cdots \tilde{x_{1}} \tilde{x_{2}}}_{2 m \text { pairs }}
$$

and

$$
g_{2}=x_{0} x_{1} \underbrace{\overline{x_{1}} \overline{x_{2}} \cdots \tilde{x_{1}} \tilde{x_{2}}}_{2 m \text { pairs }} x_{1} .
$$

By using Lemma 3 and Lemma 4, from (2) we obtain that $g_{1} \equiv(-1)^{m} g_{0}$ and $g_{2}=2^{k}(-1)^{m} g_{0}$. This implies $\alpha g_{0}+\beta^{k}(-1)^{m} g_{0} \equiv 0$.

From (3), by applying Lemma 3 and Lemma 4 , we obtain $2^{k} \alpha g_{0}+\beta g_{0} \equiv 0$. Since $\alpha \beta \neq 0$ it follows that $g_{0} \equiv 0(\bmod . \operatorname{Id}(\mathcal{W}))$. By Proposition $5, c_{n}^{Q}(\mathcal{W})=$ 0 , for all $n \geq 4 m+2$. Moreover, as in the proof of Proposition 4 it follows that $c_{n+1}(\mathcal{W}) \leq(n+1) c_{n}^{Q}(\mathcal{W})$. Hence $c_{n+1}(\mathcal{W})=0$ and so $\mathcal{W}$ is nilpotent.

\section{References}

[1] A. Berele and A. Regev, Applications of Hook Young diagrams to P.I. algebras, Journal of Algebra 82 (1983),559-567.

[2] N. L. Biggs, Discrete Mathematics, Clarendon Press, Oxford, 1989.

[3] V. Drensky, Free Algebras and PI-Algebras, Graduate Course in Algebra, Springer, Singapore, 2000.

[4] A. Giambruno and M. Zaicev, On codimension growth of finitely generated associative algebras, Advances in Mathematics 140 (1998), 145-155.

[5] A. Giambruno and M. Zaicev, Exponential codimension growth of P.I. algebras: an exact estimate, Advances in Mathematics 142 (1999), 221-243.

[6] A. Giambruno, S. Mishchenko, and M. Zaicev, Codimensions of algebras and growth functions, Advances in Mathematics 217 (2008), 1027-1052.

[7] A. Giambruno and M. Zaicev, Polynomial Identities and Asymptotic Methods, Mathematical Surveys and Monographs, Vol. 122, American Mathematical Society, Providence, RI, 2005.

[8] G James and A. Kerber, The representation theory of the symmetric group, Encyclopedia of Mathematics and its Applications, Vol. 16, Addison-Wesley, London, 1981. 
[9] S. P. Mishchenko, Varieties of linear algebras with colength one, Moscow University Mathematics Bulletin 65 (2010), 23-27.

[10] S. P. Mishchenko and A. Valenti, Varieties with at most quadratic growth, Israel Journal of Mathematics 178 (2010), 209-228.

[11] S. Mishchenko and M. Zaicev, An example of a variety of Lie algebras with a fractional exponent, Algebra, 11, Journal of Mathematical Sciences (New York) 93 (1999), 977-982.

[12] V. M. Petrogradskii, Growth of polynilpotent varieties of Lie algebras, and rapidly increasing entire functions, Matematicheskiǔ Sbornik 188 (1997), 119-138; English translation: Sbornik. Mathematics 188 (1997), 913-931.

[13] A. Regev, Existence of identities in $A \otimes B$, Israel Journal of Mathematics 11 (1972), $131-152$.

[14] M. Zaicev, Integrality of exponents of growth of identities of finite-dimensional Lie algebras, Rossiı̌kaya Akademiya Nauk. Izvestiya. Seriya Matematicheskaya 66 (2002), 23-48; English translation: Izvestiya. Mathematics 66 (2002), 463-487. 